\title{
Suicidal Ideation and Pesticide Exposure in Rural Communities of Central India
}

\author{
Prakash B. Behere ${ }^{1,2}$ Amit B. Nagdive ${ }^{2}$ Aniruddh P. Behere ${ }^{3,4}$ Richa Yadav ${ }^{4,5}$ Rouchelle Fernandes ${ }^{2}$ \\ 1 Department of Psychiatry, Dnyandeo Yashwantrao Patil University, \\ Kolhapur, Maharashtra, India \\ 2 Department of Psychiatry, Jawaharlal Nehru Medical College, Datta \\ Meghe Institute of Medical Sciences (Deemed University), Sawangi,

\begin{abstract}
Address for correspondence Amit B. Nagdive, MBBS, MD, Meghe Institute of Medical Sciences (Deemed University), Wardha,
\end{abstract} \\ Department of Psychiatry, Jawaharlal Nehru Medical College, Datta \\ Maharashtra 442107, India (e-mail: amitnagdive77@gmail.com).
} Wardha, Maharashtra, India

${ }^{3}$ Department of Pediatrics and Human Development, Helen Devos Children's Hospital, Michigan State University College of Human Medicine Grand Rapids, Grand Rapids, Michigan, United States

${ }^{4}$ Department of Psychiatry, Datta Meghe Institute of Medical

Sciences (Deemed University), Wardha, Maharashtra, India

${ }^{5}$ Department of Psychiatry and Behavioural Sciences, OU College of Medicine, Oklahoma City, Oklahoma, United States

J Neurosci Rural Pract 2021;12:623-629.

\begin{abstract}
Keywords

- death wishes

- depression

- farmers

- insecticide

- organophosphorus

Background This study aims to evaluate association between suicidal ideation and pesticide exposure in rural communities of Wardha district of Central rural India compared with villagers who are not exposed to it.

Method It was a cross-sectional study done in rural community of Wardha district in Central India. About 100 farmers who were exposed to pesticides and 100 controls who were not exposed to pesticides were included. A Semistructured proforma to record sociodemographic variables and Indian version of the Self Reporting Questionnaire-20 was used.

Results Of these 200 subjects, data were available for 85 farmers in study group and 74 in control group. In the farmers group, $31 \%$ of farmers had suicidal ideas, while $8 \%$ of controls felt like ending their life.

Conclusion Measures must be taken to store pesticides at a safer place thus reducing long-term exposure. Organic farming is a better choice to avoid suicidal ideas.
\end{abstract}

\section{Introduction}

India has $16 \%$ of the world population but is able to sustain only $2.4 \%$ of land resources. Two-thirds of population in India depend on agriculture for its livelihood and agriculture provides employment to approximately $57 \%$ of its total population. ${ }^{1}$ Despite farming being considered as an easygoing way of life, the agricultural sector in India experiences the highest number of suicides in comparison to all other industries. $^{2}$

Wardha district lies in Vidarbha region of Maharashtra state in the central part of India. Farmers in Vidarbha region grow cotton as their major crop and the region has approximately 3.4 million cotton farmers. According to the web site of Vidarbha Jan Jyoti Andolan Samiti (the Farmers Support Network), 95\% of these farmers are struggling with massive debts. published online September 10, 2021
DOI https://doi.org/

10.1055/s-0041-1735242. ISSN 0976-3147. (c) 2021. Association for Helping Neurosurgical Sick People. All rights reserved.

This is an open access article published by Thieme under the terms of the Creative Commons Attribution-NonDerivative-NonCommercial-License, permitting copying and reproduction so long as the original work is given appropriate credit. Contents may not be used for commercial purposes, or adapted, remixed, transformed or built upon. (https://creativecommons.org/ licenses/by-nc-nd/4.0/)

Thieme Medical and Scientific Publishers Pvt. Ltd., A-12, 2nd Floor, Sector 2, Noida-201301 UP, India 
Suicides accounted for $2 \%$ of all deaths in the state of Maharashtra in contrast to $1.3 \%$ across India. ${ }^{3,4}$ According to national data (the Report of Government of Punjab, Suicide in Rural Punjab, 2009) suicide in India was 10.9/100,000 population. ${ }^{5}$ The important factors leading to suicide of farmers are poverty, bankruptcy leading to pressure from loan sharks and banks, debt, rising cost of cultivation and farming commodities, dependence on rainfall, crop failure, urbanization, lack of interest in younger generation toward farming as a profession, and, most importantly, easy availability of poisons or pesticides as a source for committing suicide. More than $70 \%$ of population in India lives in rural areas and majority of these are either primarily or secondarily dependent on agriculture for their livelihood. Approximately $58 \%$ of total working population in India still depends on agriculture for work, either as farmers or as farm laborers. Farmer suicides have also been reported from other countries of the world like the United Kingdom, Sri Lanka, Canada, and Australia. In India, over the last decade, the number of suicides has increased by $28 \%$. India is ranked second in the world with respect to number of suicides. Every seventh suicide in India is committed by a farmer. The state of Maharashtra accounts for the highest number of farmers' suicide among all other states in India and has seen an annual increase at the rate of $2.5 \%$. In our region, farmer's suicide has seen a rising trend by compound rate of $9.8 \%$ annually which is more than double. Pesticide consumption and hanging are the methods employed for suicide. Majority of people residing in rural areas are farmers with the exception of a small population working in small scale industries and local places. Organophosphate group of pesticides are used in India most commonly. Though endosulfan is used quite extensively in India, its use has been long prohibited in other countries.

Looking at other countries, suicide by ingestion of pesticide was the reason in $62 \%$ of all suicides in China. ${ }^{6,7}$ In the United Kingdom, firearms were used most commonly followed by hanging and carbon monoxide poisoning. ${ }^{8}$ Earlier studies on suicides also have reported high prevalence of pesticide use as a common method of suicide in agricultural parts of Sri Lanka (71\%), Trinidad (68\%), and Malaysia (>90\%). ${ }^{9}$ Studies have reported that in Australia, higher suicide rates were observed as compared with general population, ${ }^{10}$ whereas studies in Italy show that pesticide applicators had lower incidents of accidents and suicide. ${ }^{11}$ Exposure to pesticides has been implicated in higher suicide rates in predominantly agricultural areas of southeastern Spain as compared with other areas of similar sociodemographic and socioeconomic compositions. ${ }^{12}$ Multiple studies have demonstrated increased rates of self-poisoning in farmers mostly in Asian countries of Bangladesh, China, and India. A study conducted in Sri Lanka reported that if pesticides are stored safely in fields away from homes, it contributes in a way to decrease the rate of self-poisoning. ${ }^{13}$ Risk of selfpoisoning is higher in those households where safe storage may not be available. The World Health Organization (WHO) in 2006 also advised similar pattern of storage of pesticides safely to decrease suicides. WHO recommended the design and production of boxes with help of local communities and storage of pesticides in these boxes. Community intervention for the International Association for Suicide Prevention of WHO in 2006 recommended the boxes are to be kept safely in fields, so as to reduce the impulsive acts of self-poisoning. ${ }^{14}$ Professor Flemming Konradsen, of Copenhagen University's Department of International Health, Immunology, and Microbiology has designed one such box. ${ }^{15}$ The pesticide container is made from strong plastic. It has an outer and an inner cover, is waterproof, locked and buried in the ground. The farmers normally store their pesticides in their houses or in bags outside. This makes it easily accessible in case a family member should become suicidal. This gives exposure to pesticides as well. Instead, if the pesticides are buried in a container out in the field, it gets harder for people to get their hands on them. ${ }^{15}$

Farmers usually resort to storing their pesticides either in their homes or nearby places. A study conducted recently found that around $65 \%$ of farmers committing suicides used chemicals stored in the home in China. Most commonly used pesticides are organophosphates followed by organochlorine (endosulfan).

In India, an expert panel on the Social, Environmental, and Economic Performance of Cotton Production (SEEP) found that the national consumption of pesticides was highest in the early 1990s ( $\sim 75,000$ metric tons) and has reduced subsequently.

Organophosphorous compounds had the highest amount of usage among all groups of pesticides in India in the last decade. $^{2}$

It was found that suicide rates were higher when organophosphates were used in farming. ${ }^{12}$ It has been reported that pesticide exposure can also lead to Alzheimer's disease and Parkinson's disease. ${ }^{16}$ From a mental health point of view, many studies state that apart from suicide, it can lead to depression and anxiety. ${ }^{17,18}$ Some studies also suggest that it can be a cause of mortality ascribed to mental disorder. ${ }^{19}$ On the other hand, some studies state that research in the area of suicidal ideation and pesticide exposure has not been conclusive. ${ }^{20}$ A similar study failed to demonstrate any correlation between moderate pesticide use and suicide. ${ }^{21}$ Many farmers have also described generalized weakness that they develop following exposure during spraying, as there are several applications per crop. ${ }^{22}$

Although suicide among farmers is rampant in Wardha district as described above, its relation to pesticide exposure has never been studied in this area of Central rural India.

This study was done to find out whether any correlation exists between suicidal ideation in farmers with prior exposure to pesticides in comparison to those without exposure to pesticides.

\section{Aims}

We sought to evaluate association between suicidal ideation and pesticide exposure in the rural agricultural population of Wardha district of Central rural India in comparison to villagers without any exposure to pesticides by using data from our larger study entitled "Psychological Distress in 
Survivors of Farmers' Suicides: A Cross-sectional Comparative Study from Central Part of Rural India. ${ }^{23 "}$

\section{Methods}

\section{Study Design}

Present study is a cross-sectional comparative study.

\section{Study Setting}

Study was conducted in a rural agricultural community of Wardha district in Central India. Wardha is in the Vidarbha region of Maharashtra state. Wardha district is 1 of the 36 districts in Maharashtra state in central India and is a part of Nagpur Division. As per census of 2011, Wardha district had a population of $1,300,774$ of which male and female were 668,385 and 632,389 respectively. It has eight counties which are, Wardha, Deoli, Seloo, Arvi, Ashti, Karanja, Hinganghat, and Samudrapur. ${ }^{24}$

Ethics Committee approval was obtained from Institute Ethics Committee for Research on Human Subjects. Before participating in the study, written informed consent was obtained in local language (Marathi) from the subjects.

\section{Sample Size}

A total number of 100 farmers were included in the study who had been exposed to pesticides for more than 10 years. Age, sex-matched 100 controls who were not exposed to pesticides were included in the study for comparison.

We selected 1:1 age ( \pm 2 years), sex-matched controls who were residing in same village or nearby villages (as few persons are in the villages who are not involved in farming, therefore samples were taken from nearby villages in the same geographic location). Controls and cases were not related to each other. In a situation where eligible controls could not be identified or declined to participate in the study, adjacent lanes were visited for selection of controls. Controls were selected randomly and were screened for presence of any psychopathology by psychiatric assessment.

\section{Sampling Procedure}

Samples were randomly selected based on inclusion and exclusion criteria.

\section{Tools Used}

1. Semistructured proforma to record sociodemographic variables: this proforma was used to collect sociodemographic details, such as age, sex, marital status, education, occupation, and others, were recorded.

2. The Self-Reporting Questionnaire-20: Indian version of the Self-Reporting Questionnaire-20 (SRQ-20) was used. SRQ-20 was devised by WHO to screen for psychological distress in primary health care settings, especially in developing countries. ${ }^{25}$. WHO in 1994 stated that this scale had good validity and reliability for adults ( $\geq 15$ years), can be used both as a self-rated or interviewer-administered questionnaire and consists of 20 closed questions covering suicidal ideas. Earlier studies reported that this instrument has been widely used in a variety of settings in different countries. ${ }^{26,27}$ It has high face, content, criterion, and construct validity. Many earlier studies have validated the use of the SRQ in India. ${ }^{28-31}$ A study in India demonstrated good internal consistency and a high discriminating ability for the questionnaire, but when compared with clinical interview, questionnaire demonstrated strong positive predictive value only when sensitivity was reduced. ${ }^{32}$ In a recent study in Pune, India, sensitivity and specificity of SRQ-20 were reported to be $83 \%$ and $92 \%$, respectively, as compared against interview by psychiatrist. ${ }^{33}$

\section{Inclusion Criteria}

- Both the sexes were included.

- Farmers who had earlier attempted suicide in the past but were given timely intervention were included.

- Farmers were exposed to pesticides were included for the study group.

- Control group comprised of villagers involved in professions other than farming, who were not exposed to pesticides but belonged to same or nearby villages.

- Farmers those who had a history of prior exposure to pesticides for a period of 10 years or more were only included in the study.

\section{Exclusion Criteria}

- Farmers having history of prior psychiatric illness.

- Families where prior suicide had taken place.

- Family members who were not willing to participate.

\section{Results}

Overall, 200 subjects in study and control groups (100 in each group) were eligible for the study. Data were available for 85 farmers in the study group and 74 villagers in the control group. Overall response rate was $85 \%$ in study group and $74 \%$ in control group. The reason was either they were not willing to participate or not available during the visit.

It has been found that farmers mostly belonged to the age group between 40 and 49 years (-Fig. 1). Control group mostly belonged to the age group below 30 years of age (-Fig. 2). In the study group, $47 \%$ had marginal land below 1 hectare, 28\% of farmers had small land between 1 and 2 hectares, and $19 \%$ of farmers had semi medium land between 2 and 4 hectares. Only 6\% of farmers had medium to large land, more than 4 hectares ( - Table $\mathbf{1}$ ).

In the $\mathrm{SRQ}^{34}$ (-Table 2 ), $56 \%$ of farmers had headache in comparison to $36 \%$ of controls. Also, $71 \%$ of farmers reported low appetite in comparison to only 39\% of controls. Around $59 \%$ of farmers reported to have sleep disturbances in comparison to $20 \%$ of controls. Another significant difference was that $74 \%$ of farmers reported feeling unhappy, while only $28 \%$ of controls did so. Again, $73 \%$ of farmers reported of having lost interest in things, while only $16 \%$ of controls reported the same. In the farmers group, $54 \%$ felt worthless, while in control group, only $10 \%$ felt worthless. In the farmers group, $31 \%$ of farmers felt like ending their life or had suicidal ideas, while $8 \%$ of controls 


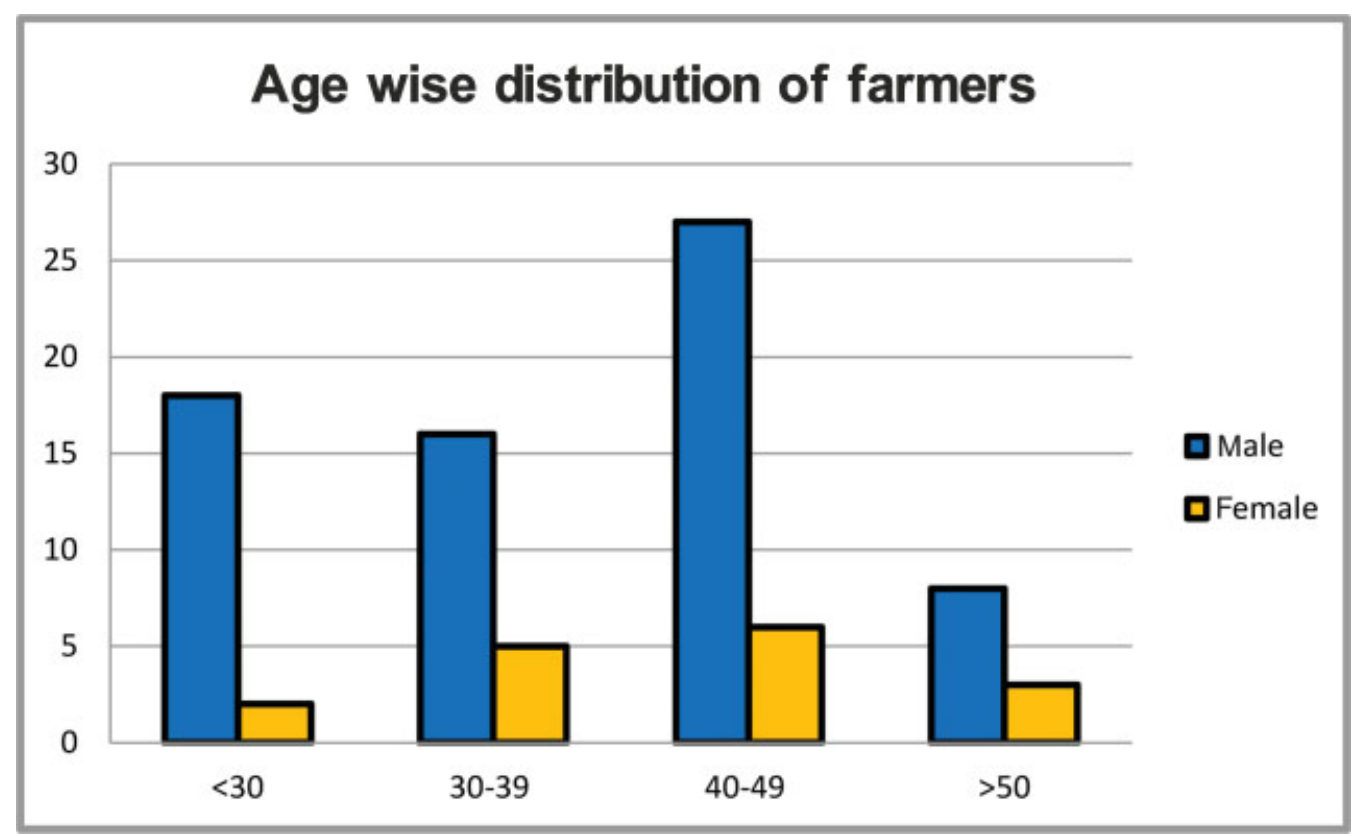

Fig. 1 Age wise distribution of farmers.

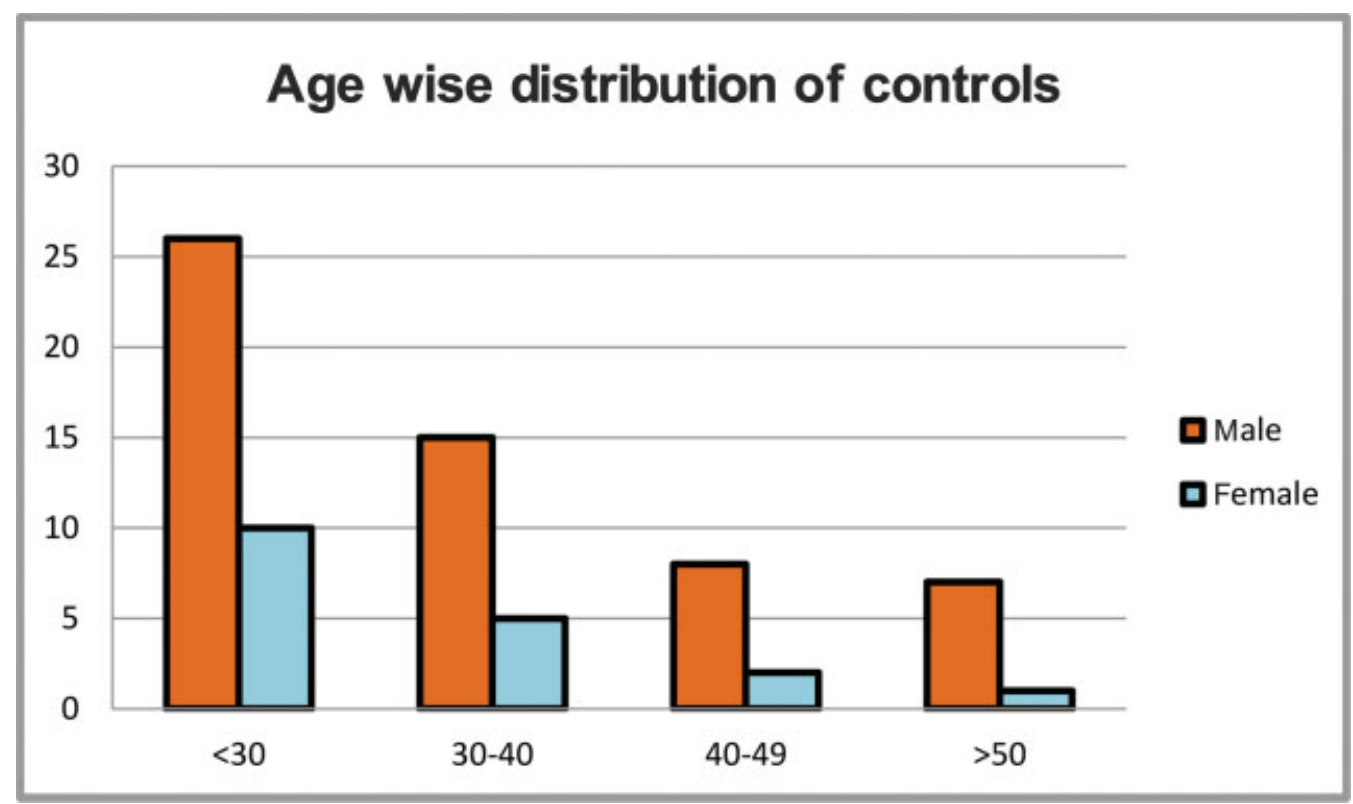

Fig. 2 Age wise distribution of control group.

Table 1 Distribution of farmers by size of land owned in hectares

\begin{tabular}{|l|l|}
\hline \multicolumn{2}{|l|}{ Land (hectares) } \\
\hline Marginal $(<1)$ & $40(47.05 \%)$ \\
\hline Small $(1-2)$ & $24(28.23 \%)$ \\
\hline Semi medium (2-4) & $16(18.82 \%)$ \\
\hline Medium-Large $(>4)$ & $05(5.88 \%)$ \\
\hline
\end{tabular}

felt like ending their life. Significantly high number of farmers around $68 \%$ felt tired all the time and 55\% reported to be tired easily. In comparison only $28 \%$ of controls felt tired all the time and $20 \%$ of controls were easily tired. Also $63 \%$ of farmers reported to feel uncomfortable in their stomach in comparison to $19 \%$ of controls.

\section{Discussion}

In our study, we found that a positive correlation existed between pesticide exposure and suicidal ideations, as $31 \%$ of farmers reported suicidal thoughts, while in the control group (who were not exposed to pesticide), only $8 \%$ reported having suicidal ideas. This finding was similar with many earlier studies on pesticide exposure and farmers suicide. 
Table 2 Responses to self-reporting questionnaire

\begin{tabular}{|c|c|c|c|c|c|c|}
\hline \multirow[t]{2}{*}{ Question no. } & \multirow[t]{2}{*}{ Questions } & \multicolumn{2}{|c|}{$\begin{array}{l}\text { Cases } \\
\text { (\% of yes } \\
\text { responses) } \\
=85\end{array}$} & \multicolumn{2}{|c|}{$\begin{array}{l}\text { Controls } \\
\text { (\% of yes } \\
\text { responses) } \\
=74\end{array}$} & \multirow[t]{2}{*}{$p$-Value } \\
\hline & & $n$ & $\%$ & $n$ & $\%$ & \\
\hline 1 & Do you often have headaches? & 47 & 55.29 & 27 & 36.49 & 0.0104 (significant) \\
\hline 2 & Is your appetite poor? & 60 & 70.59 & 29 & 39.19 & $<0.0001$ (significant) \\
\hline 3 & Do you have sleep disturbances? & 50 & 58.82 & 14 & 18.92 & $<0.0001$ (significant) \\
\hline 4 & Are you easily frightened? & 19 & 22.35 & 07 & 9.46 & 0.0180 (significant) \\
\hline 5 & Do you feel nervous, tense, or worried? & 37 & 43.53 & 18 & 24.32 & 0.0068 (significant) \\
\hline 6 & Do your hands tremble? & 29 & 34.12 & 08 & 10.81 & 0.0002 (significant) \\
\hline 7 & Is your digestion poor? & 46 & 54.12 & 23 & 31.08 & 0.0016 (significant) \\
\hline 8 & Do you have trouble thinking clearly? & 44 & 51.76 & 13 & 17.57 & $<0.0001$ (significant) \\
\hline 9 & Do you feel unhappy? & 63 & 74.12 & 21 & 28.38 & $<0.0001$ (significant) \\
\hline 10 & Do you cry more than usual? & 40 & 47.06 & 06 & 8.11 & $<0.0001$ (significant) \\
\hline 11 & Do you find it difficult to enjoy your daily activities? & 50 & 58.82 & 12 & 16.22 & $<0.0001$ (significant) \\
\hline 12 & Do you find it difficult to make a decision? & 33 & 38.82 & 11 & 14.86 & 0.0002 (significant) \\
\hline 13 & Is your daily work suffering? & 34 & 40.00 & 13 & 17.57 & 0.0010 (significant) \\
\hline 14 & Do you feel you are not usefully contributing in life? & 31 & 36.47 & 07 & 9.46 & $<0.0001$ (significant) \\
\hline 15 & Have you lost interest in things? & 62 & 72.94 & 12 & 16.22 & $<0.0001$ (significant) \\
\hline 16 & Do you feel that you are a worthless person? & 46 & 54.12 & 08 & 10.81 & $<0.0001$ (significant) \\
\hline 17 & Have you thought about ending your life? & 26 & 30.59 & 6 & 8.11 & $<0.0001$ (significant) \\
\hline 18 & Do you feel tired all the time? & 58 & 68.24 & 21 & 28.38 & $<0.0001$ (significant) \\
\hline 19 & Do you have uncomfortable feelings in your stomach? & 53 & 62.35 & 14 & 18.92 & $<0.0001$ (significant) \\
\hline 20 & Are you easily tired? & 47 & 55.29 & 15 & 20.27 & $<0.0001$ (significant) \\
\hline
\end{tabular}

Note: Bold signifies important points in drawing inference.

Many similar studies have reported that use of pesticides is associated with suicidal thoughts and behavior. ${ }^{12,17,35-38}$ Some studies, however, have found no significant relationship between suicidal ideation and pesticide exposure. Pickett et al did not find any relationship between suicide and following exposure measures: (1) the costs of purchased agricultural chemicals, (2) acres sprayed with insecticides, and (3) acres sprayed with herbicides among a group of Canadian farmers. ${ }^{39}$ Another study by Beard et al did not report any association of moderate pesticide use with suicide. $^{21}$ Easy accessibility to lethal modes like guns, pesticides, and other chemicals can also lead to suicides in farmers as reported by some studies. ${ }^{40-42}$ From our study, it was evident that $74 \%$ of the farmers who were exposed to pesticides had features of low mood or unhappiness. It was significantly lower in the control group where only $28 \%$ of controls reported of low mood. Significant number of farmers had some symptoms suggestive of depression like $72 \%$ had lost interest in things, 54\% felt worthless, $59 \%$ suffered from sleep disturbances, $71 \%$ poor appetite, $47 \%$ felt like crying more than usual, and $68 \%$ felt tired all the time. In comparison to the control group, only $16 \%$ lost interest in work, $11 \%$ felt worthless, $19 \%$ had sleep distur- bances, $39 \%$ had poor appetite, only $8 \%$ felt like crying more than usual, and $28 \%$ felt tired all the time. Thus, the difference between the study group and the control group was statistically significant. Many studies reported of farmers suffering from mental illness like depression and anxiety. ${ }^{17,43}$ Smith reported association between depressive disorder and imbalance in cholinergic pathways ${ }^{44}$ and Jaga and Dharmani stated that organophosphates, including methamidophos, inhibit cholinesterase activity. ${ }^{45}$ However, some studies suggest that many who commit suicide may not be clinically depressed at all. ${ }^{6,46}$ Conner et al reported that easy accessibility and impulsivity may lead to a completed suicide. ${ }^{47}$ Thus from our study, it seems that farmers suffered from suicidal ideas due to regular pesticide exposure. They also suffered from depression but the relation between depression and pesticide exposure has to be further studied, as there are many other variables which can lead to depression in a farmer. Can the suicidal ideation be secondary to depressive symptoms or are both independent as compared with those without insecticide exposure? It is very much likely that exposure to pesticides may have a correlation with suicidal ideation. Organic farming may play a significant role in throwing some light on this association in the future. 


\section{Limitations of Study}

This study includes the following limitations:

- Being a cross-sectional study involving one-time assessment, no inferences can be conclusively made through this study.

- Respondent bias in hope of seeking some financial gains may affect the findings, as this was the part of bigger study where farmers were expecting some compensation.

- One of the questionnaires has a strong positive predictive value only at the cost of decrease in sensitivity.

- As detailed evaluation with help of clinical interview was not done, complete assessment of the mental health status of participants was not possible.

- Needs further study in these areas as many had other depressive symptoms

In this study, suicidal ideation was found to be present in $31 \%$ of the population included in the study group (i.e., farmers with exposure to pesticide which were included in study), whereas suicidal ideation was present in only $8 \%$ of those belonging to control group sample. It was also noticed that those who had suicidal ideations also had symptoms of depression on SRQ when compared with those in control group.

\section{Conclusion}

Our study concludes that in the farmers group, $31 \%$ of farmers had suicidal ideas while $8 \%$ of controls felt like ending their life, and these were statistically significant. In view of this, measures must be taken to store pesticides at a safer place, thus reducing long-term exposure. Pesticides in the villages should be stored in a common place outside the village. Organic farming is a better choice to avoid exposure to pesticides which may avoid suicidal ideas.

\section{Implications of Study}

1. Our study mandates to take into consideration the effects of organophosphorous pesticides in farming in India and suicidal ideation.

2. Measures must be taken to store pesticides at a safer place, rather than in houses of farmers thus reducing long-term exposure.

3. The spraying of pesticides in farms should be performed by different individuals, so that the exposure to pesticides is not more than 1 hour.

4. Organic farming is a better choice to avoid suicidal ideas.

\section{Ethical Approval}

The study was granted approval by the Medical Ethics Committee of Mahatma Gandhi Institute of Medical Sciences, Wardha, Maharashtra, India. All procedures performed in this study were in accordance with the ethical standards of the institutional and/or national research committee and with the 1964 Helsinki declaration and its later amendments or comparable ethical standards. No animal or human studies were performed by the authors for this article. Informed consent was compulsorily obtained from all individual participants before inclusion in the study.

\section{Authors' Contributions}

This work was performed in collaboration with all the authors. All authors read and approved the final manuscript.

\section{Funding}

None.

\section{Conflict of Interest}

None declared.

\section{Acknowledgment}

The authors are grateful to all the farmers and villagers who participated in our study. Also, they are thankful to Dr. Debolina Chowdhury for going through the script and providing valuable suggestions.

\section{References}

1 Behere PB, Behere AP. Farmers' suicide in Vidarbha region of Maharashtra state: a myth or reality? Indian J Psychiatry 2008;50 (02):124-127

2 Abhilash PC, Singh N. Pesticide use and application: an Indian scenario. J Hazard Mater 2009;165(1-3):1-12

3 Behere PB, Bansal A. Farmers suicide in Vidarbha: everybody's concern. Journal of Mahatma Gandhi Institute of Medical Sciences 2009;14(ii):iii-v

4 Gupta PK. Pesticide exposure-Indian scene. Toxicology 2004;198 (1-3):83-90

5 Kumar P, Sharma SL. Suicides in rural Punjab. Accessed May 25, 2021 at: https://citeseerx.ist.psu.edu/viewdoc/download?doi=10. 1.1.618.9850\&rep=rep1\&type=pdf

6 Phillips MR, Li X, Zhang Y. Suicide rates in China, 1995-99. Lancet 2002;359(9309):835-840

7 Phillips MR, Yang G, Zhang Y, Wang L, Ji H, Zhou M. Risk factors for suicide in China: a national case-control psychological autopsy study. Lancet 2002;360(9347):1728-1736

8 Behere PB, Bhise MC. Farmers' suicide: across culture. Indian J Psychiatry 2009;51(04):242-243

9 Gunnell D, Eddleston M. Suicide by intentional ingestion of pesticides: a continuing tragedy in developing countries. Int J Epidemiol 2003;32(06):902-909

10 MacFarlane E, Benke G, Del Monaco A, Sim MR. Cancer incidence and mortality in a historical cohort of Australian pest control workers. Occup Environ Med 2009;66(12):818-823

11 Torchio P, Lepore AR, Corrao G, et al. Mortality study on a cohort of Italian licensed pesticide users. Sci Total Environ 1994;149(03): 183-191

12 Parrón T, Hernández AF, Villanueva E. Increased risk of suicide with exposure to pesticides in an intensive agricultural area. A 12-year retrospective study. Forensic Sci Int 1996;79(01):53-63

13 Weerasinghe M, Pieris R, Eddleston M, Hoek Wv, Dawson A, Konradsen F. Safe storage of pesticides in Sri Lanka - identifying important design features influencing community acceptance and use of safe storage devices. BMC Public Health 2008;8:276

14 World Health Organization. International Association for Suicide Prevention. Safer access to pesticides: community intervention Accessed May 25, 2021 at: https://www.who.int/mental_health/ prevention/suicide/pesticides_safer_access.pdf?ua=1 
15 Anne R. New invention helps reduce pesticide suicides. Science Nordic 2012;06:20

16 Baldi I, Lebailly P, Mohammed-Brahim B, Letenneur L, Dartigues JF, Brochard P. Neurodegenerative diseases and exposure to pesticides in the elderly. Am J Epidemiol 2003;157(05):409-414

17 Stallones L, Beseler C. Pesticide poisoning and depressive symptoms among farm residents. Ann Epidemiol 2002;12(06): 389-394

18 Stallones L. Suicide mortality among Kentucky farmers, 19791985. Suicide Life Threat Behav 1990;20(02):156-163

19 van Wijngaarden E. Mortality of mental disorders in relation to potential pesticide exposure. J Occup Environ Med 2003;45(05): 564-568

20 Zhang J, Stewart R, Phillips M, Shi Q Prince M. Pesticide exposure and suicidal ideation in rural communities in Zhejiang province, China. Bull World Health Organ 2009;87(10):745-753

21 Beard JD, Umbach DM, Hoppin JA, et al. Suicide and pesticide use among pesticide applicators and their spouses in the agricultural health study. Environ Health Perspect 2011;119(11):1610-1615

22 Cole DC, Sherwood S, Crissman C, Barrera V, Espinosa P. Pesticides and health in highland Ecuadorian potato production: assessing impacts and developing responses. Int J Occup Environ Health 2002;8(03):182-190

23 Bhise MC, Behere PB. A case-control study of psychological distress in survivors of farmers' suicides in Wardha District in central India. Int J Psych 2016;58(02):147-151

24 Wardha district: population 2011-2021 data-corona virus/ COVID data. Accessed July 17, 2021 at: https://www.census2011.co.in/census/district/342-wardha.html

25 Harding TW, de Arango MV, Baltazar J, et al. Mental disorders in primary health care: a study of their frequency and diagnosis in four developing countries. Psychol Med 1980;10(02):231-241

26 Mari JJ, Williams P. A validity study of a psychiatric screening questionnaire (SRQ-20) in primary care in the city of Sao Paulo. $\mathrm{Br}$ J Psychiatry 1986;148:23-26

27 Sen B, Williams P. The extent and nature of depressive phenomenon in primary health care. Br J Psychiatry 1987; 451:406-449

28 Harding TW, Climent CE, Diop M, et al. The WHO collaborative study on strategies for extending mental health care, II: The development of new research methods. Am J Psychiatry 1983; 140(11):1474-1480

29 de Jong K, Kam Sv, Ford N, et al. Conflict in the Indian Kashmir Valley II: psychosocial impact. Confl Health 2008;2:11-15

30 Kishore J, Reddaiah VP, Kapoor V, Gill JS. Characteristics of mental morbidity in a rural primary heath centre of Haryana. Indian J Psychiatry 1996;38(03):137-142
31 Mumford DB, Saeed K, Ahmad I, Latif S, Mubbashar MH. Stress and psychiatric disorder in rural Punjab. A community survey. $\mathrm{Br} \mathrm{J}$ Psychiatry 1997; 170:473-478

32 Patel V, Araya R, Chowdhary N, et al. Detecting common mental disorders in primary care in India: a comparison of five screening questionnaires. Psychol Med 2008;38(02):221-228

33 Chincholkar SV. Use of SRQ in psychiatric epidemiolog. Indian J Community Med 2004;XXIX(04):190-191

34 World Health Organization. User's Guide to the Self Reporting Questionnaire. Accessed May 25, 2021 at: https://apps.who.int/ iris/bitstream/handle/10665/61113/WHO_MNH_PSF_94.8.pdf? sequence $=1 \&$ is Allowed $=\mathrm{y}$

35 Browning SR, Westneat SC, McKnight RH. Suicides among farmers in three southeastern states, 1990-1998. J Agric Saf Health 2008; 14(04):461-472

36 Gunderson P, Donner D, Nashold R, Salkowicz L, Sperry S, Wittman B. The epidemiology of suicide among farm residents or workers in five North Central states, 1980-1988. Am J Prev Med 1993;9:26-32

37 Meltzer H, Griffiths C, Brock A, Rooney C, Jenkins R. Patterns of suicide by occupation in England and Wales: 2001-2005. Br J Psychiatry 2008;193(01):73-76

38 Miller K, Burns C. Suicides on farms in South Australia, 19972001. Aust J Rural Health 2008;16(06):327-331

39 Pickett W, King WD, Faelker T. Suicides among Canadian farm operators. Chronic Dis Can 1999;20(03):105-110

40 Booth N, Briscoe M, Powell R. Suicide in the farming community: methods used and contact with health services. Occup Environ Med 2000;57(09):642-644

41 Goldney RD. A global view of suicidal behaviour. Emerg Med (Fremantle) 2002;14(01):24-34

42 Gregoire A. The mental health of farmers. Occup Med (Lond) 2002;52(08):471-476

43 Salvi RM, Lara DR, Ghisolfi ES, Portela LV, Dias RD, Souza DO. Neuropsychiatric evaluation in subjects chronically exposed to organophosphate pesticides. Toxicol Sci 2003;72(02):267-271

44 Smith CUM. Elements of Molecular Neurobiology, 2nd ed.. Chichester, NY: Wiley; 1996

45 Jaga K, Dharmani C. The interrelation between organophosphate toxicity and the epidemiology of depression and suicide. Rev Environ Health 2007;22(01):57-73

46 Li XY, Phillips MR, Wang YP, Zhang C, Ji HY, Bian QT. Comparison of impulsive and non-impulsive attempted suicide.] (in Chinese). Chinese Journal of Nervous System and Mental Disorder 2003; 29:27-31

47 Conner KR, Phillips MR, Meldrum S, Knox KL, Zhang Y, Yang G. Lowplanned suicides in China. Psychol Med 2005;35(08):1197-1204 